\title{
Genetic linkage maps for Asian and American lotus constructed using novel SSR markers derived from the genome of sequenced cultivar
}

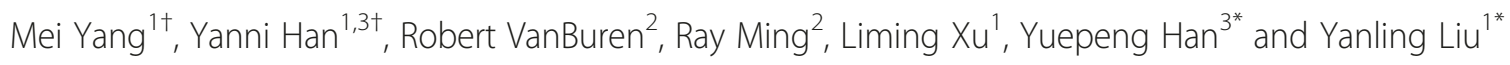

\begin{abstract}
Background: The genus Nelumbo Adans. comprises two living species, N. nucifera Gaertan. (Asian lotus) and N. lutea Pers. (American lotus). A genetic linkage map is an essential resource for plant genetic studies and crop improvement but has not been generated for Nelumbo. We aimed to develop genomic simple sequence repeat (SSR) markers from the genome sequence and construct two genetic maps for Nelumbo to assist genome assembly and integration of a genetic map with the genome sequence.

Results: A total of 86,089 SSR motifs were identified from the genome sequences. Di- and tri-nucleotide repeat motifs were the most abundant, and accounted for $60.73 \%$ and $31.66 \%$ of all SSRs, respectively. AG/GA repeats constituted $51.17 \%$ of dinucleotide repeat motifs, followed by AT/TA (44.29\%). Of 500 SSR primers tested, 386 (77.20\%) produced scorable alleles with an average of 2.59 per primer, and 185 (37.00\%) showed polymorphism among two parental genotypes, $N$. nucifera 'Chinese Antique' and N. lutea 'AL1', and six progenies of their $F_{1}$ population. The normally segregating markers, which comprised 268 newly developed SSRs, 37 previously published SSRs and 53 sequence-related amplified polymorphism markers, were used for genetic map construction. The map for Asian lotus was 365.67 cM with 47 markers distributed in seven linkage groups. The map for American lotus was $524.51 \mathrm{cM}$, and contained 177 markers distributed in 11 genetic linkage groups. The number of markers per linkage group ranged from three to 34 with an average genetic distance of $3.97 \mathrm{cM}$ between adjacent markers. Moreover, 171 SSR markers contained in linkage groups were anchored to 97 genomic DNA sequence contigs of 'Chinese Antique'. The 97 contigs were merged into 60 scaffolds.

Conclusion: Genetic mapping of SSR markers derived from sequenced contigs in Nelumbo enabled the associated contigs to be anchored in the linkage map and facilitated assembly of the genome sequences of 'Chinese Antique'. The present study reports the first construction of genetic linkage maps for Nelumbo, which can serve as reference linkage maps to accelerate characterization germplasm, genetic mapping for traits of economic interest, and molecular breeding with marker-assisted selection.
\end{abstract}

Keywords: Genetic linkage map, Genome sequence, Nelumbo, SRAP, SSR

\footnotetext{
*Correspondence: yphan@wbgcas.cn; liuyanling@wbgcas.cn

${ }^{\dagger}$ Equal contributors

${ }^{3}$ Key Laboratory of Plant Germplasm Enhancement and Specialty Agricultrue, Wuhan Botanical Garden, Chinese Academy of Sciences, Wuhan, Hubei 430074, China

${ }^{1}$ Key Laboratory of Aquatic Plant and Watershed Ecology, Wuhan Botanical Garden, Chinese Academy of Sciences, Wuhan, Hubei 430074, China

Full list of author information is available at the end of the article
} 


\section{Background}

The genus Nelumbo Adans. comprises two living species, N. nucifera Gaertn. (distributed in Asia, Australia and Russia) and N. lutea Pers. (restricted to eastern and southern North America) [1,2]. The two species differ in features of their external morphologies, such as plant size, leaf shape, petal shape and color [1,2], and have significant genetic differences as revealed by DNA markers [3-7]. The species share the same chromosome number $(2 n=16)$ and there is no interspecific reproductive barrier [8]. In Asia, lotus has been cultivated as a food crop for its rhizomes and seeds and as an ornamental plant for its magnificent flowers, in addition to its propagation for reasons related to its cultural and religious significance.

Although lotus is an important ornamental plant and a commercial crop, knowledge of the genomic constitution of Nelumbo species is limited. However, such information is a prerequisite for the identification of molecular markers linked to agronomic traits to facilitate breeding of improved lotus cultivars. A genetic linkage map is a powerful research tool for studies on plant genetics. Such a map may provide insights into genome organization, evolution and comparative genomics with related species [9]. From the perspective of improving crops, a genetic map is useful for map-based gene cloning, analysis of quantitative trait loci (QTL) underlying traits of economic importance, and molecular breeding using marker-assisted selection (MAS) [10,11]. Several DNA marker types, including random amplified polymorphic DNA (RAPD) [12-14], inter simple sequence repeats (ISSRs) [15,16], simple sequence repeats (SSRs) $[3,6,7]$ amplified fragment length polymorphisms (AFLPs) $[4,5]$, and sequence-related amplified polymorphisms (SRAPs) [17], have been developed for Nelumbo. The high degree of genetic diversity with Nelumbo revealed by these DNA markers facilitates the development of genetic linkage maps. Linkage maps have been constructed for many species, but as yet no genetic map has been developed for Nelumbo.

Simple sequence repeats are tandem repeats of one to six nucleotides present in all eukaryotic genomes [18]. Given their codominant inheritance, high polymorphism, and abundant distribution throughout genomes, SSRs have been used widely for genetic mapping, comparative analysis, and QTL analysis in plants [19,20]. Extensive efforts have been made to develop SSR markers in Nelumbo through genomic library screening using probes that contain repeated motifs and expressed sequence tags (ESTs) [3,5-7,21-23]. However, the total number of currently developed SSR markers (123 genomic SSRs and 39 EST-SSRs) is insufficient for genetic analysis in lotus. Moreover, such experimental approaches to developing SSR markers are laborious, time-consuming and costly. Therefore, there is a strong demand for additional SSR markers for lotus genome research and breeding. With the ever-increasing number of DNA sequences available in public databases, genomic sequences provide a more rapid and economic method for develop SSR markers. Based on SSRs developed from the genome sequence, high-density genetic linkage maps can be constructed [24-27].

Recently, we sequenced the genome of an ancient lotus cultivar, 'Chinese Antique,' at $60 \times$ coverage using a shotgun sequencing strategy, and the DNA sequences were assembled into 43,197 contigs. The total length of all contigs was $804 \mathrm{Mb}$, which represented $86.5 \%$ of the estimated $929 \mathrm{Mb}$ lotus genome [28]. The availability of lotus genomic sequences provides an excellent opportunity to survey SSR motifs at a genome-wide level. The SSRs developed from the contig sequences can anchor corresponding contigs onto a genetic map and further establish direct links between genetic, physical, and sequence-based maps $[25,29,30]$. Therefore, a SSR-based reference genetic map is essential for assembly of the Nelumbo genome sequences and for integration of the genetic and physical maps of Nelumbo.

In the study reported herein, we identified SSRs from the contig sequences of the lotus cultivar 'Chinese Antique' and developed a new set of SSR markers to construct two genetic linkage maps. Our objectives were to: (1) assess the distribution of SSRs in the Nelumbo genome; (2) develop a large number of SSR markers and evaluate SSR polymorphism between $N$. nucifera 'Chinese Antique' and N. lutea 'AL1'; (3) construct SSR-based reference linkage maps for Asian lotus and American lotus; and (4) anchor the SSR-associated contigs to the genetic map and facilitate assembly of the contig sequences.

\section{Results}

Identification and distribution of SSRs in the genome

A total of 86,089 SSR motifs with a minimum of five contiguous repeating units were identified within the contig sequences. Of the total SSRs identified, di- and tri-nucleotide repeat motifs were the most abundant repeat types, with frequencies of $60.73 \%$ and $31.66 \%$, respectively (Table 1). Tetra- and penta-nucleotide repeats were the least frequent repeat types (5.77\% and $1.21 \%$ ). The number of each major SSR type identified within the lotus genome is summarized in Table 1. The most abundant dinucleotide motifs were AG/GA (31.08\%) and AT/TA (26.90\%). However, rare CG/GC motifs were identified. Among trinucleotide repeats, the AAG/AGA/GAA motif was the most common (11.79\%), followed by the AAT/ATA/TAA (10.70\%) and ATG/GAT/TGA (4.05\%) motifs. GC-rich trinucleotide repeats were the least frequent. Of tetra-nucleotide repeats, AAAT/TAAA/ATAA/ AATA was the most abundant, accounting for $26.47 \%$ of all tetra-nucleotide repeats, and followed by ATAC/TACA/ ACAT/CATA (25.32\%). Among pentanucleotide repeats, 
Table 1 Distributions of the major SSR motifs identified from the genome of Nelumbo nucifera 'Chinese Antique'

\begin{tabular}{|c|c|c|c|c|c|}
\hline Motif & Number & Frequency (\%) & Range (bp) & Primers designed & Proportion (\%) \\
\hline Dinucleotide & 52282 & 60.73 & $16-110$ & 44824 & 61.20 \\
\hline$A G / G A$ & 26753 & 31.08 & $16-98$ & 23084 & 31.52 \\
\hline AT/TA & 23158 & 26.90 & $16-110$ & 19656 & 26.84 \\
\hline $\mathrm{AC} / \mathrm{CA}$ & 2367 & 2.75 & $16-72$ & 2081 & 2.84 \\
\hline $\mathrm{CG} / \mathrm{GC}$ & 4 & 0.00 & $16-20$ & 3 & 0.00 \\
\hline Trinucleotide & 27258 & 31.66 & $15-141$ & 22745 & 31.05 \\
\hline AAG/AGA/GAA & 10146 & 11.79 & $15-141$ & 8668 & 11.83 \\
\hline AAT/ATA/TAA & 9212 & 10.70 & $15-102$ & 7049 & 9.62 \\
\hline ATG/GAT/TGA & 3485 & 4.05 & $15-84$ & 3158 & 4.31 \\
\hline AAC/ACA/CAA & 1160 & 1.35 & $15-90$ & 962 & 1.31 \\
\hline AGG/GAG/GGA & 1099 & 1.28 & $15-48$ & 958 & 1.31 \\
\hline other & 2156 & 2.50 & $15-138$ & 1950 & 2.66 \\
\hline Tetranucleotide & 4964 & 5.77 & 20-184 & 4276 & 5.84 \\
\hline AAAT/TAAA/ATAA/AATA & 1314 & 1.53 & $20-164$ & 1190 & 1.62 \\
\hline ATAC/TACA/ACAT/CATA & 1257 & 1.46 & $20-184$ & 1071 & 1.46 \\
\hline TATC/ATCT/TCTA/CTAT & 858 & 1.00 & 20-84 & 741 & 1.01 \\
\hline TाТ/TTCT/TCTT/CTTा & 740 & 0.86 & $20-60$ & 596 & 0.81 \\
\hline TTAA/TAAT/AATT/ATTA & 179 & 0.21 & 20-36 & 164 & 0.22 \\
\hline Other & 616 & 0.72 & $20-144$ & 514 & 0.70 \\
\hline Pentanucleotide & 1045 & 1.21 & $25-145$ & 964 & 1.32 \\
\hline AGAAG/GAAGA/AAGAG/AGAGA/GAGAA & 253 & 0.29 & $25-80$ & 211 & 0.29 \\
\hline AAAAG/AAAGA/AAGAA/AGAAA/GAAAA & 109 & 0.13 & $25-45$ & 106 & 0.14 \\
\hline TाTCC/TTCCT/TCCTT/CCTT/СТTC & 104 & 0.12 & $25-145$ & 102 & 0.14 \\
\hline AAAGG/AAGGA/AGGAA/GGAAA/GAAAG & 98 & 0.11 & $25-70$ & 90 & 0.12 \\
\hline ATATA/TATAA/ATAAT/TAATA/AATAT & 95 & 0.11 & $25-95$ & 90 & 0.12 \\
\hline Other & 386 & 0.45 & $25-80$ & 365 & 0.50 \\
\hline
\end{tabular}

The SSR motifs represent variants of both strands of the DNA sequence (e.g., AAT/ATA/TAA includes the reverse complements ATT, TTA and TAT).

AGAAG/GAAGA/AAGAG/AGAGA/GAGAA motif was more common than other combinations. These data reflected AG/GA and AT/TA repeat motifs were the most abundant SSRs in the $N$. nucifera genome.

\section{Development of SSR markers and detection of polymorphism}

All microsatellites were selected for SSR marker development. From the 86,089 SSR-containing sequences in contigs, 73,246 non-redundant SSR primer pairs were identified. Priority was given to di- and tri-nucleotide repeats, which accounted for $61.20 \%$ and $31.05 \%$ of the total number of SSR primers, respectively. Among the markers that contained dinucleotide repeats, the largest proportion was for AG/GA, followed by AT/TA motifs (Table 1). Only three markers that contained CG/GC motifs were identified because this motif was very rare in the genome sequences analyzed. Of the markers for trinucleotide repeats, the AAG/AGA/GAA motif was the most common (11.83\%), followed by the AAT/ATA/ TAA (9.62\%) and ATG/GAT/TGA (4.31\%) motifs.

Only 500 pairs of primers were tested for their amplification potential in the two parents and their six $\mathrm{F}_{1}$ progenies. Primer sequence information, repeat motifs, amplicon sizes and polymorphism features for these 500 primers are listed in Additional file 1. Among these primers, 459 amplified at least one fragment in the genetic materials, and 73 primers that produced ambiguous fragments or smears were excluded from the genotype analysis of the $\mathrm{F}_{1}$ lines. Of the successfully amplified primers (386 pairs), the number of alleles per primer ranged from one to seven with a mean of 2.59 , and a majority of them amplified two (218 pairs of primers) or three ( 82 pairs of primers) alleles. For 386 pairs of primers, 185 showed polymorphism among all eight lines, and 54 showed polymorphism in the two parents but no segregation in the six lines of the $F_{1}$ population. Finally, 175 SSR primers were used to analyze the genotypes of all $F_{1}$ progenies, which produced 450 markers with an average of 2.57 per primer (Table 2). 
Table 2 Markers generated for the genetic mapping

\begin{tabular}{lllllll}
\hline Marker & $\begin{array}{l}\text { Number of primer } \\
\text { used }\end{array}$ & $\begin{array}{l}\text { Number of polymorphic } \\
\text { primer }\end{array}$ & $\begin{array}{l}\text { Maternal } \\
\text { markers }\end{array}$ & $\begin{array}{l}\text { Paternal } \\
\text { markers }\end{array}$ & $\begin{array}{l}\text { Total } \\
\text { markers }\end{array}$ & $\begin{array}{l}\text { Number of distorted } \\
\text { marker }\end{array}$ \\
\hline Novel SSR & 500 & 175 & 202 & 248 & 450 & 182 \\
Previous SSR & 95 & 33 & 27 & 41 & 68 & 31 \\
SRAP & 32 & 28 & 18 & 73 & 91 & 21 \\
Total & 627 & 238 & 247 & 362 & 609 & 234 \\
\hline
\end{tabular}

\section{Analysis of genotypes in the $F_{1}$ population}

For 450 markers identified using the 175 novel SSR primers, $202(44.89 \%)$ segregated in the female parent and 248 (55.11\%) segregated in the male parent (Table 2). Thirty-three out of 95 previously published SSR primers were polymorphic among the parents and six $F_{1}$ progenies (Additional file 2), which produced 68 segregating markers in all seedlings of the mapping population. The average number of markers per primer was 1.93. Among these 68 SSR markers, 27 (39.71\%) segregated in the female parent and $41(60.29 \%)$ segregated in the male parent (Table 2).

Of the 32 SRAP primers tested, 28 reproducibly amplified clear polymorphic fragments in the parents and all $F_{1}$ progenies. A total of 91 SRAP markers were identified in the mapping population (Table 2). On average, there were 3.25 markers per primer combination, and the number of markers ranged from one to eight. Of the 91 SRAP markers, 18 (19.78\%) originated from the female parent and the remaining of 73 SRAP markers $(80.22 \%)$ originated from the male parent.

The goodness-of-fit of observed-to-expected allelic ratios was analyzed using the $\chi^{2}$ test. Of the total of 609 markers, 234 (38.42\%) showed a significant deviation from Mendelian expectations $(P<0.05) .129$ markers deviated toward the female parent and 105 markers toward the male parent. The 129 SSR distorted markers derived from the female parent showed no segregation in the $F_{1}$ progenies, which indicated a high level of homozygosity in the female parent. The 234 distorted markers were excluded from the linkage analysis because they could lead to false linkage between some markers or linkage groups. The remaining 375 markers, which comprised 118 maternal markers and 257 paternal markers, showed the expected 1:1 segregation ratio and were used to establish the linkage groups.

\section{Construction of two linkage maps}

A framework map was constructed using 305 SSR markers (Additional file 3). Only 32 markers were identified in the maternal map and were assigned to eight small linkage groups (LG1-F to LG8-F) with a total length of $362.52 \mathrm{cM}$. The number of markers per linkage group varied from three to seven. In contrast, 137 SSR markers were assigned to the paternal map, which consisted of eight major linkage groups (LG1-M to LG8-M) and three triplets (LG9-M to
LG11-M) that covered a total length of $495.37 \mathrm{cM}$. The number of markers in LG1-M to LG8-M varied from eight to 27 with an average of 12.8 (Additional file 3).

To increase map coverage, SRAP markers were added to the framework map to construct an integrated map. A total of 375 markers, including 268 novel SSR markers, 37 previously published SSR markers, and 70 SRAP markers were available for map construction. The addition of SRAP markers to the framework map allowed the SSR markers that were originally unlinked or floating to be placed as accessory markers. Using the SSR marker orders in the framework map as the preferred orders, 47 markers composed of 36 SSR and 11 SRAP markers were identified in the maternal map. These were assigned to seven linkage groups (LG1-F to LG7-F), with three to 18 markers in each group. The map spanned $365.67 \mathrm{cM}$ with an average interval of $7.78 \mathrm{cM}$ between adjacent markers (Table 3 and Figure 1). The paternal linkage map consisted of 177 markers distributed over 11 linkage groups (LG1-M to LG11-M). There were from three to 34 markers per linkage group. The paternal map spanned $524.51 \mathrm{cM}$ with an average interval of $2.96 \mathrm{cM}$ between adjacent markers. The genetic length of each linkage group varied from $3.14 \mathrm{cM}$ (LG11-M) to 98.45 cM (LG1-M) (Table 3 and Figure 2).

\section{Discussion}

In the study, we analyzed the frequency of microsatellites identified from 43,197 contig sequences of $N$. nucifera 'Chinese Antique'. The $804 \mathrm{Mb}$ of genomic sequence analyzed covers $86.5 \%$ of the lotus genome. One SSR was detected in every $9.33 \mathrm{~kb}$ in the genome sequences. The frequency of SSRs in lotus is much lower than that reported in the genome sequences of Brassica [24,29,31,32] and rice [33], but higher than the estimated frequency of SSRs in the genome of sorghum [27]. In the contig sequences, dinucleotide repeat motifs $(60.73 \%)$ were the most frequently detected, followed by tri- (31.66\%), tetra- (5.77\%) and penta-nucleotide (1.21\%) motifs. The most abundant dinucleotide motif detected was the AG/GA type (31.08\%), followed by AT/TA (26.90\%) and AC/CA (2.75\%). The most common trinucleotide motif detected was the AAG/ AGA/GAA type, followed by AAT/ATA/TAA and ATG/ GAT/TGA (Table 1). These motif types and their proportions in the lotus genome are in close agreement with the 
Table 3 Number of markers and the length per linkage group of the integrated map, and the number of contigs corresponded to every linkage group

\begin{tabular}{|c|c|c|c|c|c|c|}
\hline \multirow[t]{2}{*}{ Linkage group } & \multicolumn{4}{|c|}{ Number of markers } & \multirow{2}{*}{$\begin{array}{l}\text { Total length } \\
\text { (cM) }\end{array}$} & \multirow{2}{*}{$\begin{array}{l}\text { Number } \\
\text { of contig }\end{array}$} \\
\hline & Novel SSR & Previous SSR & SRAP & Total & & \\
\hline \multicolumn{7}{|c|}{ Female (Asian lotus) map } \\
\hline LG1-F & 9 & 1 & 8 & 18 & 111.34 & 9 \\
\hline LG2-F & 5 & 2 & 1 & 8 & 78.14 & 4 \\
\hline LG3-F & 5 & 1 & 2 & 8 & 27.16 & 5 \\
\hline LG4-F & 4 & 0 & 0 & 4 & 62.68 & 2 \\
\hline LG5-F & 2 & 1 & 0 & 3 & 30.8 & 2 \\
\hline LG6-F & 3 & 0 & 0 & 3 & 30.52 & 1 \\
\hline LG7-F & 2 & 1 & 0 & 3 & 25.02 & 2 \\
\hline \multicolumn{7}{|c|}{ Male (American lotus) map } \\
\hline LG1-M & 20 & 5 & 9 & 34 & 98.46 & 19 \\
\hline LG2-M & 16 & 0 & 3 & 19 & 49.89 & 12 \\
\hline LG3-M & 14 & 4 & 5 & 23 & 80.02 & 12 \\
\hline LG4-M & 7 & 1 & 9 & 17 & 62.15 & 4 \\
\hline LG5-M & 11 & 2 & 2 & 15 & 58.49 & 6 \\
\hline LG6-M & 17 & 1 & 3 & 21 & 51.58 & 11 \\
\hline LG7-M & 16 & 2 & 4 & 22 & 46.67 & 13 \\
\hline LG8-M & 8 & 2 & 4 & 14 & 34.76 & 5 \\
\hline LG9-M & 3 & 0 & 2 & 5 & 30.72 & 2 \\
\hline LG10-M & 2 & 1 & 1 & 4 & 8.62 & 3 \\
\hline LG11-M & 2 & 1 & 0 & 3 & 3.14 & 3 \\
\hline Total & 146 & 25 & 53 & 224 & 890.16 & 97 \\
\hline
\end{tabular}

patterns observed in dicots such as Arabidopsis [34], Brassica $[24,31,32]$ and papaya [35], and in the monocot sorghum [27], in which AT and AG combinations of base pair motif types are predominant. The distribution of SSRs in the lotus genome is different from those observed in humans and Drosophila, in which AC is the most frequent dinucleotide repeat motif, followed by AT and AG [18]. The GC repeat motif is extremely rare in eukaryotic genomes, except in rice [33,34], and was absent from the lotus genome. These data suggested that AG and AT motifs were rich in the lotus genome.

The pseudo-testcross strategy is suitable for construction of genetic maps using a $F_{1}$ population and was applied first for genetic mapping in Eucalyptus [36]. Given that lotus is protogynous and cross-pollinated, a high level of heterozygosity is predicted for the genomes of Nelumbo species. With this prediction in mind, we created a $F_{1}$ mapping population to construct genetic maps of Nelumbo in the present study. The two parents, $N$. nucifera 'Chinese Antique' and $N$. lutea 'AL1', diverge strongly in their geographical distributions and important morphological traits, such as plant size, and shape and color of the leaves and petals. The two parents also show considerable genetic differences [3-7], which was confirmed here by the high polymorphism (61.91\%) detected using the novel SSR markers (Additional file 1). Of 609 markers identified in the mapping population, the markers originated from the female parent $(40.56 \%)$ were less than those inherited from the male parent (59.44\%).

234 markers $(38.42 \%)$ showed distorted segregation at the $P<0.05$ level (Table 2), which was higher than the distorted segregation ratio reported for Dendrobium [37], strawberry [38] and ryegrass [39]. Segregation distortion is a common phenomenon in mapping studies with a $F_{1}$ population [37-39]. Distortion of segregation ratios may result from biological factors, such as genetic isolation mechanisms, chromosome loss, locus duplication, and gamete selection $[40,41]$. Nonbiological factors, such as scoring errors and sampling errors, also can lead to distortion in segregation ratios $[42,43]$. In the present study, both biological factors and technical problems may have caused the observed segregation distortion in the $F_{1}$ population. The high level of homozygosity of the female parent 'Chinese Antique', which was unexpected, has contributed to the considerable segregation distortion in the $F_{1}$ population. Hence, 129 of the markers with distorted segregation ratios derived from the female parent showed no segregation in the $F_{1}$ progenies. Our 
recent analysis of the $\mathrm{F}_{1}$ population estimate heterozygosity to be $0.03 \%$ for 'Chinese Antique', and 0.37 for 'AL1' (unpublished data). Thus, the similarly low heterozygosity in the male parent aggravated the distorted segregation in the $F_{1}$ population. We observed that 96 markers skewed to the male parent showed similar segregation ratios and were distributed in a group spanning $20.23 \mathrm{cM}$ (data not shown).

Molecular marker selection for genetic mapping is crucial for the credibility of linkage maps. Due to their inherited characteristics, SSR markers have many advantages in genetic mapping. They can also serve as anchor markers during comparative mapping with related species [20]. However, the high level of homozygosity of the female parent precluded the availability of SSR markers assigned to the maternal map. Moreover, using a single type of DNA marker to construct a lotus linkage map would easily lead to uneven marker distribution and large intervals between adjacent markers. Therefore, in order to increase the coverage of the linkage map and reduce the gap between markers, SRAP markers were selected to analyze the genotypes of the $F_{1}$ population. Given that SRAP primers usually amplify the genomic intron and exon regions of functional genes [44], they complement the use of SSR markers. When SRAP markers were added to the framework map constructed using SSR markers, the total length of linkage groups increased from 857.89 to $890.16 \mathrm{cM}$, and the average interval between two adjacent markers decreased from 5.08 to $3.97 \mathrm{cM}$. Moreover, SSR markers in LG4-F and LG6-F of the maternal framework map were incorporated into one linkage group LG1-F in the integrated map. No large gaps $(>25 \mathrm{cM}$ ) were detected in these genetic linkage maps (Additional file 3 and Additional file 4). Thus, to some extent, the application of SSR and SRAP markers increased the length of the linkage map and reduced the distance between two adjacent markers.

Using normally segregating markers, genetic linkage maps for Nelumbo were constructed successfully (Figures 1 


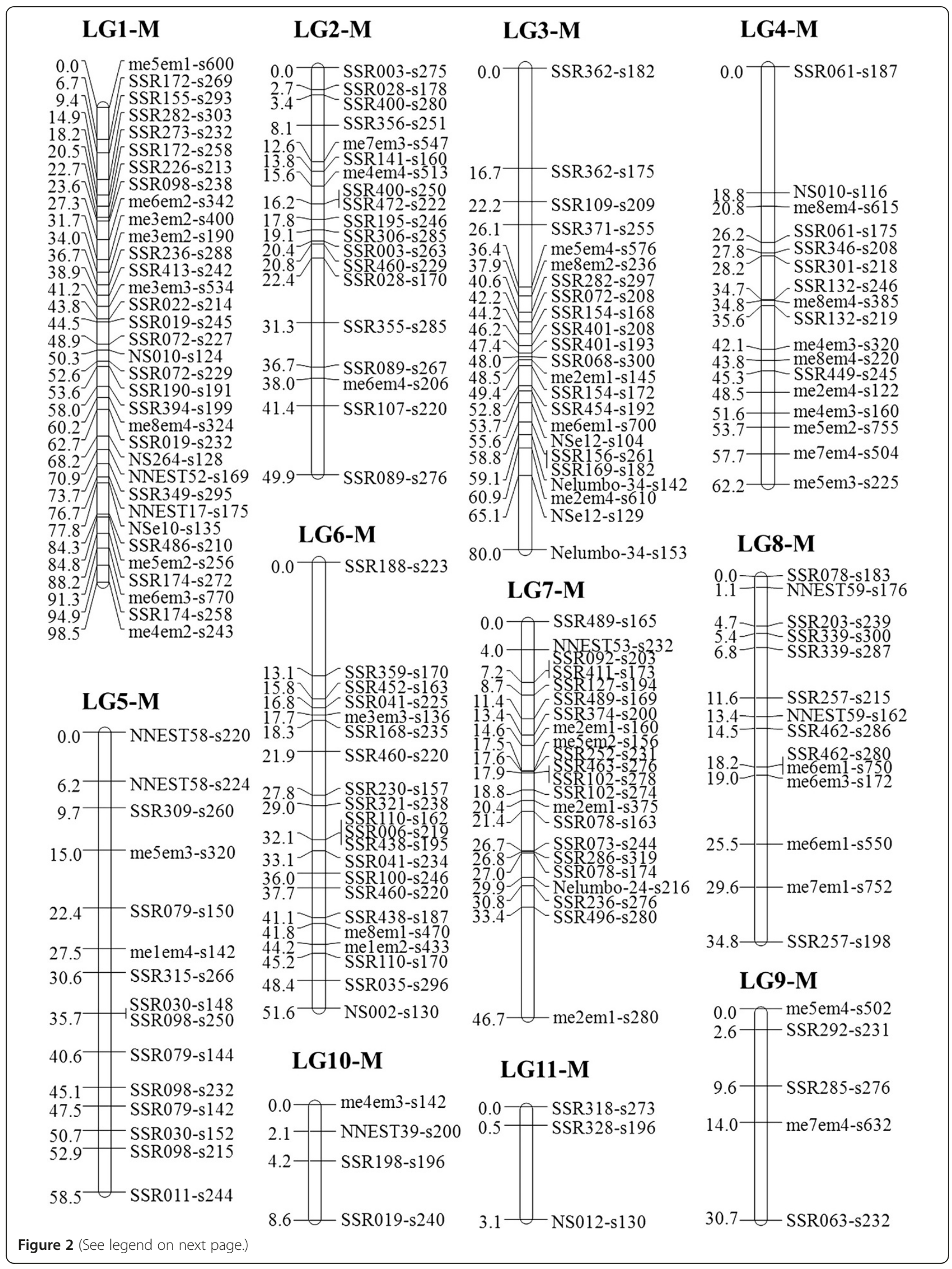


(See figure on previous page.)

Figure 2 Genetic linkage map of American lotus (Nelumbo lutea) constructed with SSR and SRAP markers. Map distances in centi-

Morgans (CM) and marker names are shown on the left and right sides of each linkage group (LG1-M to LG11-M), respectively. The marker nomenclature corresponds to the primer name followed by " $s$ " (abbreviation of "size") and the size of product (in base pair).

and 2). The integrated linkage maps comprised 171 SSR and 53 SRAP markers. These SSR markers were derived from 93 novel SSR primers and 20 previously published SSR primers, and were anchored to 97 contigs of 'Chinese Antique' (Table 3 and Additional file 4). The contig-based SSR markers can anchor corresponding contigs onto a genetic map and further establish direct links between genetic, physical, and sequence-based maps [25,29,30]. This SSR-based linkage maps are important reference linkage maps with which to anchor contigs and assemble the genome sequence of Nelumbo. As a result, these 97 contigs were merged into 60 scaffolds (Additional file 4). In the following genome assembly work, on the basis of the order of SSR markers in linkage groups and single nucleotide polymorphism (SNP) markers identified by restriction-site associated DNA sequencing (RAD-seq) technology, 43,197 contigs of lotus were assembled into approximately 3,605 sequence scaffolds (unpublished data).

In theory, the number of linkage groups should be consistent with the number of haploid chromosomes. As a genus comprising only diploid species, members of Nelumbo have eight pairs of chromosomes. In the present study, 7 and 11 linkage groups were detected for the female and male plants, respectively, based on SSR and SRAP markers (Table 3). Thus, the numbers of linkage groups did not match with the haploid chromosome number. It may be inferred that some linkage groups in the paternal map should correspond to one chromosome, but were divided because a large interval existed between the linkage group. No markers were detected in the eighth linkage group used to create the maternal map. This may be attributable to the low degree of heterozygosity in the maternal genome. Many preliminary genetic maps for other plant species usually contain a higher number of linkage groups than expected $[37,45]$. Because the mapping population used in the present study was the $F_{1}$ generation of the cross between 'Chinese Antique' and 'AL1', no recombination between homoeologous chromosomes was possible [46]. Therefore, the markers in the two parental linkage groups could not integrate into one group. The high quality of the DNA markers and the accuracy of genetic mapping were exemplified by the clear separation of linkage groups for 'Chinese Antique' and 'AL1' (Figures 1 and 2).

To improve the linkage map, the number of male and female linkage groups should be supposed equal to the haploid chromosome number, and the density of linkage group should be increased. The use of more advanced genotyping technology, such as high-throughput sequencing for SNP discovery at the whole-genome or -transcriptome scale, would aid the construction of a complete genetic linkage map. RAD-seq of multiple individuals using Illumina technology can identify and score thousands of SNP markers randomly distributed across the target genome $[47,48]$. The platform can effectively generate dense linkage maps for QTL analysis [49-51]. Our recent work involving the RAD-seq approach identified 6,622 SNPs in the present $F_{1}$ mapping population and enabled construction of a high-density genetic map together with the SSR markers developed in the present study. This paternal map spanned $494.3 \mathrm{cM}$ and comprised 4,031 markers (3,895 SNP and 136 SSR) in nine linkage groups (unpublished data). Using the common SSR markers as anchor markers, comparative analysis identified the collinearity of these linkage maps. The genetic maps generated in the present study can serve as reference linkage maps of Nelumbo species for efficient studies of comparative genetics, QTL analysis of traits of economic importance, and molecular breeding with MAS.

\section{Conclusions}

We here present the first report of the construction of genetic linkage maps of Nelumbo with SSR markers derived from sequenced contigs. A total of 73,246 nonredundant SSR markers were identified at a genomewide level. Of 500 SSR primers that were selected, 185 (37.00\%) showed polymorphism among the two parental genotypes and six progenies of their $F_{1}$ population. Using the normally segregating markers, two genetic maps for Asian and American lotus were constructed, which comprised 224 markers that spanned $890.16 \mathrm{cM}$. Ninety-seven contigs were anchored to linkage groups and were integrated into 60 scaffolds. The genetic maps will accelerate the exploitation of genetic resources, QTL analysis, map-based gene cloning, and molecular breeding with MAS in Nelumbo.

\section{Methods}

\section{Plant materials}

A segregating $F_{1}$ population derived from a cross between $N$. nucifera 'Chinese Antique' (female) and $N$. lutea 'AL1' (male) was used to construct a linkage map. The segregating population consisted of 51 seedlings. Young leaves of these seedlings and their parents were collected for DNA extraction using the cetyltrimethylammonium bromide (CTAB) method described by Doyle and Doyle 
[52]. DNA samples were diluted to $50 \mathrm{ng} \mu \mathrm{L}^{-1}$, and stored at $-20^{\circ} \mathrm{C}$ until use.

\section{Mining of microsatellites from the contig sequences and primer design}

A total of 43,197 sequenced contigs of 'Chinese Antique' were screened for SSR motifs with the Msatfinder script implemented in PERL (http://www.genomics.ceh.ac.uk/ msatfinder). All SSRs with repeat motifs of two or more base pairs and greater than five repeating units were recorded.

All microsatellites were analyzed for SSR marker development. To eliminate redundancy and to avoid designing redundant sets of primers for the same locus, the selected SSR-containing sequences were screened for redundancy by comparisons among the primer sequences and by means of BLASTN analysis against genomic sequences. The resultant contigs were parsed to design primer pairs, employing the standalone Primer3 (http://frodo. wi.mit.edu/) program with a MISA-generated Primer3 input file [53]. The primer length was set between 18 and 23 nucleotides with an optimum size of 20 nucleotides. The melting temperatures ranged from $50^{\circ} \mathrm{C}$ to $70^{\circ} \mathrm{C}$ with an optimum temperature of $55^{\circ} \mathrm{C}$. The optimum GC content was set to $50 \%$ with a minimum of $30 \%$ and a maximum of $70 \%$. The predicted PCR products ranged from 100 to $300 \mathrm{bp}$.

\section{Evaluation of SSR polymorphism}

A total of 500 primers were selected from newly designed SSR markers and used to evaluate SSR polymorphism. Primers were designated 'SSR'. Their sequences are listed in Additional file 1. All primers were synthesized by Invitrogen Biotech (Shanghai, China). All of the 500 SSRs, together with 95 previously published SSRs, were used to detect polymorphism among six $\mathrm{F}_{1}$ progenies and their parents. The previously published primers prefixed by 'Nelumbo,' 'NSh', 'PR' and 'NS' were derived from genomic sequences of $N$. nucifera [6,21-23]. Primer pairs prefixed 'NNEST' and 'NSe' were developed from EST sequences by Pan et al. [7] and Kubo et al. [6]. The polymorphic SSR markers were applied for subsequent confirmation in all $F_{1}$ lines.

All PCR amplifications were conducted in a $10-\mu \mathrm{L}$ reaction mixture that contained $50 \mathrm{ng}$ of DNA, $10 \times$ PCR buffer, $2.0 \mathrm{mmol} \mathrm{L}^{-1} \mathrm{Mg}^{2+}, 0.2 \mathrm{mmol} \mathrm{L}{ }^{-1}$ dNTPs, $0.8 \mathrm{mmol} \mathrm{L}^{-1}$ of each primer, and $0.5 \mathrm{U}$ Taq DNA polymerase (MBI Fermentas Burlington, Ontario, Canada). A Bio-Rad MyCycler Thermal Cycler (Bio-Rad, California, USA) and the following program were used: initial denaturation at $94^{\circ} \mathrm{C}$ for $5 \mathrm{~min} ; 35$ cycles of $40 \mathrm{~s}$ at $94^{\circ} \mathrm{C}$, $30 \mathrm{~s}$ at the appropriate annealing temperature, and $40 \mathrm{~s}$ of extension at $72^{\circ} \mathrm{C}$; and a final elongation step at $72^{\circ} \mathrm{C}$ for $10 \mathrm{~min}$
All PCR products were separated in a $6 \%$ denaturing polyacrylamide gel that contained $7 \mathrm{~mol} \mathrm{~L}^{-1}$ urea and $0.5 \times$ Tris-borate-EDTA (TBE) electrophoretic buffer. The gel was pre-run in $0.5 \times$ TBE buffer at $75 \mathrm{~W}$ constant power for $30 \mathrm{~min}$. After the samples had been loaded, the gel was run until the xylene cyanol tracking dye had passed through two-thirds of the gel. After electrophoresis, the gel was stained with silver nitrate solution. Allele sizes were compared with a 50-bp DNA Step Ladder (Promega, Madison, WI, USA). SSR markers were named according to SSR primer names followed by the sizes (in base pair) of the DNA fragments scored.

\section{Genotyping analysis by SRAP marker}

Combination of the previously published ME forward primers (ME1 to ME8) and EM reverse primers (EM1 to EM4) for sequence-related amplified polymorphism (SRAP) marker were used to analyze the genotypes of the $\mathrm{F}_{1}$ population [44]. Each 10- $\mu \mathrm{L}$ PCR mixture consisted of

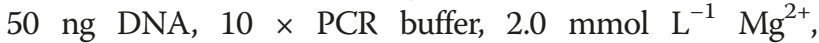
$0.2 \mathrm{mmol} \mathrm{L}^{-1} \mathrm{dNTPs}, 0.8 \mathrm{mmol} \mathrm{L}^{-1}$ of each primer, and 0.75 U Taq DNA polymerase. The PCR amplification procedure was as follows: $5 \mathrm{~min}$ of denaturation at $94^{\circ} \mathrm{C}$; five cycles of $1 \mathrm{~min}$ of denaturation at $94^{\circ} \mathrm{C}, 1 \mathrm{~min}$ of annealing at $35^{\circ} \mathrm{C}$, and $1 \mathrm{~min}$ of elongation at $72^{\circ} \mathrm{C}$, then 30 cycles with the annealing temperature at $50^{\circ} \mathrm{C}$; and a final elongation step of $7 \mathrm{~min}$ at $72^{\circ} \mathrm{C}$. The amplified SRAP fragments were separated and visualized using the same procedure for the SSR fragments.

\section{Segregation analysis and construction of genetic linkage map}

The genotypic data for the $F_{1}$ population analyzed with SSR and SRAP markers were used for the construction of a genetic map of lotus. The linkage analysis was performed using JoinMap version 4.0 [54]. Two heterozygous alleles from either parent were expected to segregate at a 1:1 ratio. The observed and expected allelic ratios for all markers were compared using the chi-squared $\left(\chi^{2}\right)$ test. Markers were excluded from the linkage analysis when they showed significantly distorted segregation $(P<0.05)$. Only the markers with a $P$ value higher than 0.05 were used for further linkage analysis.

To construct a suitably robust map, we followed the strategy described by Yang et al. [55]. This involved first using SSR markers to construct a preliminary framework map, and then joining SRAP markers to construct an integrated map using the order of the SSR markers in the framework as preferred orders (the 'fixed order' function). During construction of the framework and integrated maps, maternal and paternal data sets were created using the function 'Create Maternal and Paternal Population Node' in the JoinMap program. Maternal and paternal data were used to construct maternal and 
paternal maps, respectively. The regression mapping algorithm was used for map construction. The threshold for goodness of fit was set to $\leq 5.0$ with logarithm of the odds ratio (LOD) scores $>1.0$ and a combination frequency $<$ 0.35. Map distances were calculated using Kosambi's mapping function, and denoted in centi-Morgans (cM).

\section{Additional files}

\section{Additional file 1: Primer sequence information, repeat motifs, amplicon sizes, and polymorphism features for novel simple sequence repeat (SSR) markers from the genome sequences of Nelumbo nucifera 'Chinese Antique'.}

Additional file 2: Details on previously published simple sequence repeat (SSR) markers of Nelumbo nucifera.

Additional file 3: Framework map constructed using simple sequence repeat (SSR) markers for Nelumbo.

Additional file 4: Integrated map constructed using simple sequence repeat (SSR) and sequence-related amplified polymorphism (SRAP) markers for Nelumbo.

\section{Competing interests}

The authors declare that there are no competing interests.

\section{Authors' contributions}

MY carried out genetic mapping, analyzed the data, and drafted the manuscript. YNH participated in the marker development and polymorphism detected. RVB participated in the sequence analyses and the marker development. RM participated in the design of the study and helped draft the manuscript. LMX cultivated and provided the plant materials. YPH participated in genetic mapping and drafted the manuscript. YLL conceived the study, participated in its design and coordination, and helped to draft the manuscript. All authors read and approved the final manuscript.

\section{Acknowledgments}

This research was financially supported by Knowledge Innovation Project of Chinese Academy of Sciences (KSCX2-EW-J-20) and National Natural Science Foundation of China (31272195).

\section{Author details}

${ }^{1}$ Key Laboratory of Aquatic Plant and Watershed Ecology, Wuhan Botanical Garden, Chinese Academy of Sciences, Wuhan, Hubei 430074, China. ${ }^{2}$ Department of Plant Biology, University of Illinois at Urbana-Champaign, Urbana, IL 61801, USA. ${ }^{3}$ Key Laboratory of Plant Germplasm Enhancement and Specialty Agricultrue, Wuhan Botanical Garden, Chinese Academy of Sciences, Wuhan, Hubei 430074, China.

Received: 21 May 2012 Accepted: 7 November 2012

Published: 21 November 2012

\section{References}

1. Wang QC, Zhang XY: Colored Illustration of Lotus Cultivars in China. Beijing: China Forestry Publishing House; 2005.

2. Zhang XY, Chen LQ, Wang QC: New lotus flower cultivars in China. Beijing: China forestry Publishing House; 2011.

3. Pan L, Quan ZW, Hu JH, Wang GY, Liu SN, He Y, Ke WD, Ding Y: Genetic diversity and differentiation of lotus (Nelumbo nucifera) accessions assessed by simple sequence repeats. Ann Appl Biol 2011, 159(3):428-441.

4. Fu J, Xiang $Q$, Zeng $X$, Yang $M$, Wang $Y$, Liu $Y$ : Assessment of the genetic diversity and population structure of lotus cultivars grown in China by amplified fragment length polymorphism. J Am Soc Hortic Sci 2011, 136(5):339-349.

5. Hu J, Pan L, Liu H, Wang S, Wu Z, Ke W, Ding Y: Comparative analysis of genetic diversity in sacred lotus (Nelumbo nucifera Gaertn.) using AFLP and SSR markers. Mol Biol Rep 2012, 39(4):3637-3647.

6. Kubo N, Hirai M, Kaneko A, Tanaka D, Kasumi K: Classification and diversity of sacred and American Nelumbo species: the genetic relationships of flowering lotus cultivars in Japan using SSR markers. Plant Genetic Resources 2009, 7(03):260-270.

7. Pan L, Xia Q, Quan Z, Liu H, Ke W, Ding Y: Development of novel EST-SSR from sacred Lotus (Nelumbo nucifera Gaertn) and their utilization for the genetic diversity analysis of $N$. nucifera. J Hered 2010, 101(1):71-82

8. Huang $X Q$, Chen JY, Huang GC: Preliminary studies on biosystematical relationship between the two Nelumbo species. Acta Horticulture Sinica 1992, 19(2):164-170.

9. Shibaike $\mathrm{H}$ : Molecular genetic mapping and plant evolutionary biology. J Plant Res 1998, 111(3):383-388.

10. Collard B, Jahufer $M$, Brouwer J, Pang E: An introduction to markers, quantitative trait loci (QTL) mapping and marker-assisted selection for crop improvement: The basic concepts. Euphytica 2005, 142(1-2):169-196.

11. Collard BCY, Mackill DJ: Marker-assisted selection: an approach for precision plant breeding in the twenty-first century. Philosophical Transactions of the Royal Society B:Biological Sciences 2008, 363(1491):557-572.

12. Guo HB, Li S, Peng J, Ke W: Genetic diversity of Nelumbo accessions revealed by RAPD. Genet Resour Crop Evol 2007, 54(4):741-748.

13. Li Z, Liu X, Gituru RW, Juntawong N, Zhou M, Chen L: Genetic diversity and classification of Nelumbo germplasm of different origins by RAPD and ISSR analysis. Sci Hortic 2010, 125(4):724-732.

14. Na A, Guo HB, Ke WD: Genetic variation in rhizome lotus (Nelumbo nucifera Gaertn. ssp. nucifera) germplasms from China assessed by RAPD markers. Agricultural Sciences in China 2009, 8(1):31-39.

15. Chen YY, Zhou RC, Lin XC, Wu KQ, Qian XE, Huang SZ: ISSR analysis of genetic diversity in sacred lotus cultivars. Aquatic Botanty 2008, 89(3):311-316.

16. Han YC, Teng CZ, Wahiti G, Zhou MQ, Hu ZL, Song YC: Mating system and genetic diversity in natural populations of Nelumbo nucifera (Nelumbonaceae) detected by ISSR markers. Plant Systematics and Evolution 2009, 277(1):13-20.

17. Yang M, Han YN, Xu LM, Zhao JR, Liu YL: Comparative analysis of genetic diversity of lotus (Nelumbo) using SSR and SRAP markers. Sci Hortic 2012, 142:185-195.

18. Katti MV, Ranjekar PK, Gupta VS: Differential distribution of simple sequence repeats in eukaryotic genome sequences. Mol Biol Evol 2001, 18(7):1161-1167.

19. Park YJ, Lee JK, Kim NS: Simple sequence repeat polymorphisms (SSRPs) for evaluation of molecular diversity and germplasm classification of minor crops. Molecules 2009, 14(11):4546-4569.

20. Varshney RK, Graner A, Sorrells ME: Genic microsatellite markers in plants: features and applications. Trends Biotechnol 2005, 23(1):48-55.

21. Kubo N, Hirai M, Kaneko A, Tanaka D, Kasumi K: Development and characterization of simple sequence repeat (SSR) markers in the water lotus (Nelumbo nucifera). Aquatic Botanty 2009, 90(2):191-194.

22. Pan L, Quan Z, Li S, Liu H, Huang X, Ke W, Ding Y: Isolation and characterization of microsatellite markers in the sacred lotus (Nelumbo nucifera Gaertn). Mol Ecol Notes 2007, 7(6):1054-1056.

23. Tian HL, Chen XQ, Wang JX, Xue JH, Wen J, Mitchell G, Zhou SL: Development and characterization of microsatellite loci for lotus (Nelumbo nucifera). Conserv Genet 2008, 9(5):1385-1388.

24. Li HT, Chen X, Yang Y, Xu JS, Gu JX, Fu J, Qian XJ, Zhang SC, Wu JS, Liu KD: Development and genetic mapping of microsatellite markers from whole genome shotgun sequences in Brassica oleracea. Mol Breed 2011, 28(4):585-596

25. Thudi M, Bohra A, Nayak SN, Varghese N, Shah TM, Penmetsa RV, Thirunavukkarasu N, Gudipati S, Gaur PM, Kulwal PL, et al: Novel SSR markers from BAC-end sequences, DArT arrays and a comprehensive genetic map with 1,291 marker loci for chickpea (Cicer arietinum). PLOS One 2011, 6(11):e27275.

26. Geethanjali S, Kadirvel P, Pe AR, Rao E, Wang JF: Development of tomato SSR markers from anchored BAC clones of chromosome 12 and their application for genetic diversity analysis and linkage mapping. Euphytica 2011, 178(2):283-295.

27. Yonemaru J, Ando T, Mizubayashi T, Kasuga S, Matsumoto T, Yano M: Development of genome-wide simple sequence repeat markers using whole-genome shotgun sequences of sorghum (Sorghum bicolor (L.) Moench). DNA Res 2009, 16(3):187-193.

28. Diao Y, Chen L, Yang GX, Zhou MQ, Song YC, Hu ZL, Liu JY: Nuclear DNA C-values in 12 species in nymphaeales. Caryologia 2006, 59(1):25-30.

29. Xu JS, Qian XJ, Wang XF, Li RY, Cheng XM, Yang YA, Fu J, Zhang SC, King $\mathrm{GJ}, \mathrm{Wu} J \mathrm{~S}$, et al: Construction of an integrated genetic linkage map for 
the A genome of Brassica napus using SSR markers derived from sequenced BACs in B. rapa. BMC Genomics 2010, 11:594.

30. Han Y, Zheng D, Vimolmangkang S, Khan MA, Beever JE, Korban SS: Integration of physical and genetic maps in apple confirms wholegenome and segmental duplications in the apple genome. J Exp Bot 2011, 62(14):5117-5130.

31. Iniguez-Luy F, Voort A, Osborn T: Development of a set of public SSR markers derived from genomic sequence of a rapid cycling Brassica oleracea L. genotype. Theor Appl Genet 2008, 117(6):977-985.

32. Cheng XM, XU JS, Xia S, Gu JX, Yang Y, Fu J, Qian XJ, Zhang SC, Wu JS, Liu $\mathrm{K}$ : Development and genetic mapping of microsatellite markers from genome survey sequences in Brassica napus. Theor Appl Genet 2009, 118(6):1121-1131.

33. Temnykh S, DeClerck G, Lukashova A, Lipovich L, Cartinhour S, McCouch S: Computational and experimental analysis of microsatellites in rice (Oryza sativa L.): frequency, length variation, transposon associations, and genetic marker potential. Genome Res 2001, 11(8):1441-1452.

34. Lawson MJ, Zhang LQ: Distinct patterns of SSR distribution in the Arabidopsis thaliana and rice genomes. Genome Biol 2006, 7:R14.

35. Wang J, Chen C, Na J-K, Yu Q, Hou S, Paull R, Moore P, Alam M, Ming R: Genome-wide comparative analyses of microsatellites in papaya. Tropical Plant Biology 2008, 1(3):278-292.

36. Grattapaglia D, Sederoff R: Genetic linkage maps of Eucalyptus grandis and Eucalyptus urophylla using a pseudo-testcross: mapping strategy and RAPD Markers. Genetics 1994, 137(4):1121-1137.

37. Lu JJ, Zhao HY, Suo NN, Wang S, Shen B, Wang HZ, Liu JJ: Genetic linkage maps of Dendrobium moniliforme and D. officinale based on EST-SSR, SRAP, ISSR and RAPD markers. Sci Hortic 2012, 137:1-10.

38. Sargent DJ, Passey T, Surbanovski N, Lopez Girona E, Kuchta P, Davik J, Harrison R, Passey A, Whitehouse AB, Simpson DW: A microsatellite linkage map for the cultivated strawberry (Fragaria $\times$ ananassa) suggests extensive regions of homozygosity in the genome that may have resulted from breeding and selection. Theor Appl Genet 2012, 124(7):1229-1240.

39. Jones EJ, Dupal MD, Dumsday JD, Hughes LH, Forster JF: An SSR-based genetic linkage map for perennial ryegrass (Lolium perenne L.). Theor Appl Genet 2002, 105(4):577-584.

40. Liebhard R, Koller B, Gianfranceschi L, Gessler C: Creating a saturated reference map for the apple (Malus $\times$ domestica Borkh.) genome. Theor Appl Genet 2003, 106(8):1497-1508.

41. Moyle LC, Graham EB: Genome-wide associations between hybrid sterility QTL and marker transmission tatio distortion. Mol Biol Evol 2006, 23(5):973-980.

42. Devey ME, Fiddler TA, Liu BH, Knapp SJ, Neale DB: An RFLP linkage map for loblolly pine based on a three-generation outbred pedigree. Theor Appl Genet 1994, 88(3):273-278.

43. Echt CS, Nelson CD: Linkage mapping and genome length in eastern white pine (Pinus strobus L.). Theor Appl Genet 1997, 94(8):1031-1037.

44. Li G, Quiros CF: Sequence-related amplified polymorphism (SRAP), a new marker system based on a simple PCR reaction: its application to mapping and gene tagging in Brassica. Theor Appl Genet 2001, 103(2):455-461.

45. Varshney RK, Bertioli DJ, Moretzsohn MC, Vadez V, Krishnamurthy L, Aruna R, Nigam SN, Moss BJ, Seetha K, Ravi K, et al: The first SSR-based genetic linkage map for cultivated groundnut (Arachis hypogaea L.). Theor Appl Genet 2009, 118(4):729-739.

46. Pearl HM, Nagai C, Moore PH, Steiger DL, Osgood RV, Ming R: Construction of a genetic map for arabica coffee. Theor Appl Genet 2004, 108(5):829-835.

47. Davey JW, Blaxter ML: RADSeq: next-generation population genetics. Briefings in Functional Genomics 2010, 9(5-6):416-423.

48. Davey JW, Hohenlohe PA, Etter PD, Boone JQ, Catchen JM, Blaxter ML: Genome-wide genetic marker discovery and genotyping using nextgeneration sequencing. Nat Rev Genet 2011, 12(7):499-510.

49. Pfender W, Saha M, Johnson E, Slabaugh M: Mapping with RAD (restrictionsite associated DNA) markers to rapidly identify QTL for stem rust resistance in Lolium perenne. Theor Appl Genet 2011, 122(8):1467-1480.

50. Scaglione D, Acquadro A, Portis E, Tirone M, Knapp SJ, Lanteri S: RAD tag sequencing as a source of SNP markers in Cynara cardunculus L. BMC Genomics 2012, 13:3.

51. Barchi L, Lanteri S, Portis E, Acquadro A, Vale G, Toppino L, Rotino GL: Identification of SNP and SSR markers in eggplant using RAD tag sequencing. BMC Genomics 2011, 12:304.
52. Doyle JL, Doyle JJ: Isolation of plant DNA from fresh tissue. Focus 1990, 12:13-15.

53. Rozen S, Skaletsky H: Primer3 on the WWW for General Users and for Biologist Programmers. 1999, 132:365-386.

54. JW VO: JoinMap ${ }^{\circledR}$ 4.0: software for the calculation of genetic linkage maps in experimental populations. Wageningen, Netherlands: Kyazma BV; 2006.

55. Yang M, Ding G, Shi L, Feng J, Xu F, Meng J: Quantitative trait loci for root morphology in response to low phosphorus stress in Brassica napus. Theor Appl Genet 2010, 121(1):181-193.

doi:10.1186/1471-2164-13-653

Cite this article as: Yang et al:: Genetic linkage maps for Asian and American lotus constructed using novel SSR markers derived from the genome of sequenced cultivar. BMC Genomics 2012 13:653.

\section{Submit your next manuscript to BioMed Central and take full advantage of:}

- Convenient online submission

- Thorough peer review

- No space constraints or color figure charges

- Immediate publication on acceptance

- Inclusion in PubMed, CAS, Scopus and Google Scholar

- Research which is freely available for redistribution
C Biomed Central 